\title{
ARTÍCULOS
}

Sometido 02.03.2016. Aprobado 29.08.2016

Evaluado por el sistema double blind review. Editor Científico: Francisco Rondan-Cataluña

DOI: http://dx.doi.org/10.1590/So034-759020170203

\section{COMPROMISO, RECURSOS, EMPRENDIMIENTO EXPORTADOR Y RESULTADOS EMPRESARIALES}

\author{
Compromisso, recursos, empreendimento exportador e resultados empresariais \\ Commitment, resources, export enterprise and business results
}

\section{RESUMEN}

La presente investigación tiene un doble objetivo. En primer lugar, partiendo de las premisas del enfoque basado en los recursos (Resource-based view [RBV]), conocer cómo determinados factores internos (recursos y compromiso exportador) pueden influir en el nivel de emprendimiento que muestra la empresa exportadora en los mercados exteriores. En segundo lugar, conocer el efecto del emprendimiento exportador en los resultados empresariales. Las relaciones propuestas quedan plasmadas en un modelo conceptual que se contrastará a partir de una muestra multisectorial de 212 empresas exportadoras españolas. Los resultados revelan que: a) el emprendimiento exportador depende positivamente del compromiso exportador y de los recursos asociados a la experiencia y a la estructura; b) se corrobora el efecto positivo que el nivel de emprendimiento exportador genera en los resultados empresariales, por lo que es recomendable que las empresas exportadoras desarrollen procesos de internacionalización acelerados, con presencia en múltiples países simultáneamente y con elevada intensidad exportadora.

PALABRAS CLAVE | Emprendimiento exportador, compromiso exportador, recursos, resultado exportador, mercados exteriores.

\section{RESUMO}

A presente pesquisa tem dois objetivos. Em primeiro lugar, partindo das premissas da visão baseada em recursos (Resource-based view [RBV]), saber como determinados fatores internos (recursos e compromisso exportador) podem influir no nível de empreendedorismo que a empresa exportadora apresenta nos mercados externos. Em segundo lugar, saber o efeito do empreendedorismo exportador nos resultados empresariais. As relações propostas refletem-se claramente num modelo conceitual que será confirmado a partir de uma amostra multissetorial de 212 empresas exportadoras espanholas. Os resultados revelam que: a) o empreendedorismo exportador depende positivamente do compromisso exportador e dos recursos associados à experiência e à estrutura; b) corrobora-se o efeito positivo que o nível de empreendedorismo exportador gera nos resultados empresariais, sendo recomendável que as empresas exportadoras desenvolvam processos de internacionalização acelerados, com presença em múltiplos países simultaneamente e com alta intensidade exportadora.

\section{anavarro@us.es}

Profesor de la Universidad de Sevilla, Facultad de Ciencias Económicas y

Empresariales - Sevilla, España

\section{MANUEL REY-MORENO}

mrmoreno@us.es

Profesor de la Universidad de Sevilla,

Facultad de Turismo y Finanzas -

Sevilla, España

\section{RAMÓN BARRERA-BARRERA}

rbarrera@us.es

Profesor de la Universidad de Sevilla,

Facultad de Ciencias Económicas y

Empresariales - Sevilla, España
PALAVRAS-CHAVE / Empreendedorismo exportador, compromisso exportador, recursos, resultado exportador, mercados exteriores.

\section{ABSTRACT}

The current research has two aims. Firstly, starting from the premises of the Resource-Based View-(RBV) to know how specific internal factors (resources and export commitment) can influence an exporting firm's level of entrepreneurship in foreign markets. Secondly, to find out what the effect of export entrepreneurship is on business results. The relationships proposed are clearly reflected in a conceptual model which will be verified from a multisectorial sample of 212 Spanish exporting firms. The results reveal that: a) export entrepreneurship positively depends on export commitment and the resources associated with experience and structure; $b$ ) there is a positive effect of the level of export entrepreneurship on business results. It is recommended that export firms develop accelerated internationalization processes, be present in numerous countries simultaneously and have a high export intensity.

KEYWORDS / Export entrepreneurship, export commitment, resources, exportperformance, foreign markets. 


\section{INTRODUCCIÓN}

Emprendimiento y exportación son dos elementos esenciales en el proceso de crecimiento económico de los países, a través de la creación o desarrollo de nuevos negocios (Hessels, 2007). El emprendimiento contribuye al crecimiento económico a través de la generación y transmisión de conocimiento, del incremento de la competitividad y de la diversidad (Audretsch \& Keilbach, 2004). La exportación tiene un impacto positivo sobre la cantidad nacional de reservas de divisas y el aumento de la prosperidad nacional, lo que contribuye a la expansión de la industria doméstica, a la mejora de la productividad y del empleo (Hessels \& Stel, 2011). También es generadora de procesos de aprendizaje, tanto desde la óptica del capital humano como del tecnológico (Blalock \& Gertler, 2004; Yeoh, 2004).

El Gobierno de España, consciente de la importancia de ambos factores -emprendimiento y exportación-, ha establecido como línea prioritaria, dentro del Plan Estratégico de Internacionalización de la Economía Española 2014-2015, el fomento de la cultura emprendedora entre las empresas exportadoras españolas (con especial atención a las empresas exportadoras de pequeño y mediano tamaño -PYMEX-, por ser estas las más numerosas pero las que menor volumen exportador obtienen). En este contexto, el Gobierno de España considera prioritario el desarrollo de estudios que permitan conocer los antecedentes y consecuencias del emprendimiento exportador, y este es el ámbito en el cual se encuadra la presente investigación.

Ambos tópicos individualmente, emprendimiento y exportación, son recurrentes en la literatura económica, de management y de marketing. Sin embargo, es muy limitado el conocimiento existente sobre emprendimiento en la exportación (Hessels \& Stel, 2011). Su origen puede encontrarse en la fragmentación y ausencia de un marco teórico aceptado sobre el emprendimiento en los negocios internacionales, campo de estudio caracterizado por diversos gaps de conocimiento, inconsistencias teóricas y resultados contradictorios (Keupp \& Gassmann, 2009). En este contexto, la presente investigación pretende avanzar en el conocimiento del emprendimiento exportador delimitándolo conceptualmente, empleando para ello las dimensiones de grado, alcance y velocidad, así como analizar algunos de sus antecedentes internos a partir del enfoque basado en los recursos (Resource-based view [RBV]). En ello se centrará el primer objetivo de la investigación.

Por otra parte, el resultado exportador es el output fundamental sobre el que gira la toma de decisiones en la empresa exportadora (Navarro, Acedo, Losada, \& Ruzo, 2011). Diversa es la literatura en torno a este tópico de investigación, destacándose en los últimos años los trabajos centrados en el efecto que produce en la actividad exportadora la internacionalización acelerada o enfoque born global (Knight \& Liesch, 2016; Zander, McDougallCovin, \& Rose, 2015). Sin embargo, como señala Navarro-García (2016), la mayoría de estos estudios se ha centrado en una sola dimensión del comportamiento emprendedor de la empresa exportadora, la velocidad, analizando las consecuencias en los resultados de una internacionalización temprana. La adopción de esta perspectiva obvia lo que la empresa exportadora hace una vez que se internacionaliza, lo cual lleva a tener en cuenta los mercados en los que actúa, es decir, el alcance y el nivel -gradode intensidad exportadora. Este gap investigador ha abierto una nueva línea de estudio (Navarro-García, Schmidt, \& ReyMoreno, 2015) en el que encuadramos el segundo objetivo de la presente investigación, cuyo objeto es conocer la influencia del emprendimiento exportador, definido a partir de la velocidad, el grado y el alcance, en el resultado de la actividad exportadora.

Dos son las principales contribuciones del trabajo. Primero, tomando como referencia el RBV, se demuestra que el nivel de emprendimiento exportador está condicionado por factores de tipo interno, tanto personales -compromiso exportadorcomo propios de la organización -experiencia y estructura-. Segundo, se corrobora que aquellas empresas exportadoras que muestran mayor nivel de emprendimiento, plasmándose en el desarrollo de procesos de internacionalización acelerados, con presencia internacional en múltiples países y elevada intensidad exportadora, obtienen mejores resultados -cuantitativos y cualitativos- en los mercados exteriores que aquellas otras que muestran comportamientos más conservadores.

Para conseguir los objetivos propuestos, el trabajo tiene la siguiente estructura.: En primer lugar, se establece el marco conceptual, definiendo el concepto y dimensiones del emprendimiento exportador. A continuación, se modelizan algunos de sus determinantes internos empleando el RBV, lo que permite definir las hipótesis de investigación. En tercer lugar, se explica el método de investigación empleado a partir de una muestra multisectorial de 212 exportadores españoles. Posteriormente, se discuten los resultados y su importancia para la actividad exportadora española, y se ofrecen las principales conclusiones y contribuciones del estudio, tanto académicas como desde la óptica de la gestión. El trabajo finaliza con las limitaciones y sugerencias para futuras líneas de investigación.

\section{MARCO TEÓRICO Y PLANTEAMIENTO DE HIPÓTESIS}

Emprender implica crear o desarrollar un nuevo negocio, en el caso de los exportadores, en mercados diferentes al doméstico. 
Sin embargo, como señalan Yeoh y Jeong (1995), refiriéndose a la exportación, no debe confundirse emprender con emprendimiento, pues si bien lo primero simplemente puede implicar iniciar operaciones de comercio exterior, lo segundo -emprendimientoimplica hacerlo de una manera proactiva. En este sentido, estos autores señalan que las empresas exportadoras pueden diferenciarse según su orientación emprendedora. Así, mientras algunos exportadores tienden a ser proactivos, innovadores y con menor aversión al riesgo en la búsqueda de oportunidades comerciales en los mercados extranjeros, otros tienden a mostrarse reactivos o conservadores.

En línea con Yeoh y Jeong (1995), Ibeh y Young (2001) definen el emprendimiento exportador como el proceso por el cual los individuos, ya sean por sí mismos o dentro de las organizaciones, aprovechan las oportunidades de mercado -extranjero- teniendo en cuenta los recursos disponibles y los factores del entorno que los afectan. Esta definición destaca que el emprendimiento exportador depende de factores internos (ej. recursos) y externos (ej. entorno). Sin embargo, la definición de lbeh y Young (2001) viene a considerar que es un exportador emprendedor cualquier empresa que inicia actividad exportadora, planteando un importante interrogante: ¿Existen distintos niveles de emprendimiento entre las empresas que ya exportan? Al intentar responder a esta cuestión, Ibeh (2003) añade a la definición de lbeh y Young (2001) que son exportadores emprendedores aquellos que se muestran proactivos en la búsqueda de oportunidades de exportación relacionadas con innovaciones de productos-mercados. Esta definición abrió el debate sobre qué debe entenderse por proactividad en la exportación, debate que no parece haberse resuelto en la literatura sobre exportación, pues se ha centrado en las actitudes y orientaciones de los export managers y no en el comportamiento propio de la organización (Navarro et al., 2011). En el presente trabajo consideramos que este debate puede resolverse teniendo en cuenta tres aspectos clave asociados al emprendimiento en los negocios internacionales (Jones, Coviello, \& Tang, 2011; Keupp \& Gassmann, 2009): velocidad, grado y alcance, con las necesarias matizaciones asociadas a la exportación como forma de entrada en los mercados extranjeros.

La velocidad hace referencia al tiempo que la empresa tarda en iniciar su actividad exportadora (Acedo \& Jones, 2007), así como al ritmo al que la empresa exportadora crece y se desarrolla en los mercados extranjeros (Kuivalainen, Saarenketo, \& Puumalainen, 2012). En este contexto, serán más emprendedoras aquellas empresas que inician la exportación de forma temprana, normalmente en los 6 primeros años de vida, pues reflejan una clara orientación internacional (Gallego \& Casillas, 2014).
El alcance determina el número de mercados extranjeros -países- en los cuales la empresa exportadora genera ventas internacionales, referido en la literatura como equivalente a la extensión o diversificación exportadora (Beleska-Spasova, Glaister, \& Stride, 2012; Ruzo, Losada, Navarro, \& Díez, 2011). A este respecto, 10 es el número de países que se considera que deben tenerse en cuenta para discriminar cuándo una empresa exportadora está tendiendo a concentrar mercados (exporta a $\leq 10$ países) o a diversificar mercados (exporta a > 10 países), reflejando en este último caso un comportamiento más emprendedor (Ruzo et al., 2011).

El grado o intensidad exportadora determina el nivel de orientación hacia los mercados exteriores, en relación al doméstico, de la empresa exportadora (Kuivalainen, Sundqvist, \& Servais, 2007), empleándose normalmente el ratio ventas de exportación respecto a las ventas totales para su medición. Tampoco existe acuerdo en la literatura sobre qué ratio tomar para evaluar la orientación emprendedora del exportador, considerándose que $20 \%$ puede ser un buen punto de corte (Pla-Barber \& Alegre, 2007).

En este contexto, teniendo en cuenta las aportaciones señaladas en la literatura, el emprendimiento exportador puede conceptualizarse, a partir de las dimensiones de velocidad, alcance y grado, como el proceso por el cual una empresa -sus directivos-, empleando la exportación como forma de entrada a los mercados extranjeros, desarrolla una exportación temprana -en los 6 primeros años de vida-, tendiendo, independientemente de su tamaño, a comercializar sus productos y/o servicios a través de una estrategia de diversificación de mercados -suelen estar presentes simultáneamente en más de 10 países- y con una intensidad exportadora superior al $20 \%$.

\section{Compromiso y recursos como determinantes del emprendimiento exportador}

La revisión de la literatura revela la existencia de dos grupos de factores determinantes del emprendimiento exportador (Jones et al., 2011; Keupp \& Gassmann, 2009): a) internos, asociados a factores personales o individuales de quienes toman las decisiones en la exportación, así como a las características propias de la organización, sus recursos y capacidades; b) externos, asociados al entorno o sector en que la empresa exportadora actúa. En la presente investigación, centrada en los factores de tipo interno, se van a considerar: a) factores personales, cómo es la actitud de los export managers hacia la exportación, reflejada en su compromiso exportador; b) factores de tipo organizacional, de forma que a partir de los principios del RBV, se consideran 
recursos asociados a la estructura -departamento de exportacióny al aprendizaje -experiencia general e internacional-.

En este contexto, las actitudes de los export managers constituyen un elemento central en el progreso de la organización en los mercados extranjeros (Lages, Jap, \& Griffith, 2008), pudiendo influir en el grado de orientación emprendedora (Acedo \& Galán, 2011). Entre esas actitudes, las revisiones de Aaby y Slater (1989) y Zou y Stan (1998) otorgan un rol esencial al compromiso exportador. Estudios posteriores avalan estos planteamientos, tanto como antecedente directo del resultado exportador (Beamish, Karavis, Goerzen, \& Lane, 1999; Styles \& Ambler, 2000; Navarro, Losada, Ruzo, \& Díez, 2010b), como factor mediador de la relación estrategia de marketing internacionalresultado exportador (Hultman, Robson, \& Katsikeas, 2009; Lages \& Montgomery, 2004; Sousa, Martínez-López, \& Coelho, 2008; Navarro, Acedo, Losada, \& Ruzo, 2010a). Sin embargo, muy escaso es el conocimiento existente sobre la influencia directa que puede ejercer el compromiso en el emprendimiento exportador.

En el presente trabajo, se define el compromiso exportador como la actitud -voluntad- de los decisores de dedicar adecuados recursos financieros, humanos y directivos a la actividad exportadora (Donthu \& Kim, 1993; Autores, 2010a). Esta voluntad atenúa los riesgos y barreras percibidas de la exportación (Styles \& Ambler, 2000) y aumenta la predisposición a ofrecer un mayor soporte y apoyo a los distribuidores extranjeros (Cavusgil \& Zou, 1994). También facilita la adaptación del programa de marketing-mix a las necesidades de los consumidores extranjeros impulsando la diferenciación de los productos y servicios de la empresa exportadora (Alteren \& Tudoran, 2016). Todo ello potencia la orientación internacional de la organización (Machado, Nique, \& Fehse, 2016), fomentando el inicio temprano de movimientos de internacionalización y la predisposición de la empresa exportadora a estar presente en numerosos países de forma simultánea, y todo ello con una notable intensidad exportadora, traduciéndose en mayores niveles de emprendimiento exportador (Navarro-García, Rondán-Cataluña, \& Acedo-González, 2013). Estos argumentos sostienen el planteamiento de la siguiente hipótesis de investigación:

H1: El compromiso exportador influye positivamente en el nivel de emprendimiento de la empresa exportadora.

Por otra parte, el RBV concibe a los recursos como la piedra angular de los resultados empresariales (Barney, 1991). El epicentro de este enfoque es conocer cómo las empresas pueden lograr ventajas competitivas y resultados superiores a los de sus competidores en el mismo mercado, mediante la adquisición y explotación de recursos que son únicos e inimitables (Dhanaraj \& Beamish, 2003; Makadok, 2001).
La relación recursos y resultados ha centrado la atención de los investigadores en el ámbito de la actividad exportadora (Cadogan, Kuivalainen, \& Sundqvist, 2009; Colton, Roth, \& Bearden, 2010; Lages, Silva, \& Styles, 2009; Morgan, Kaleka, \& Katsikeas, 2004; Morgan, Vorhies, \& Schlegelmilch, 2006; Singh, 2009). Sin embargo, es amplio el desconocimiento que existe sobre la relación recursos-emprendimiento exportador (Jones et al., 2011). En la presente investigación, siguiendo las pautas establecidas en el trabajo de Ruzo et al. (2011) vamos a considerar dos tipos de recursos a) recursos asociados a la experiencia y b) recursos asociados a la estructura.

En relación a la experiencia, distinguimos entre experiencia general e internacional. La experiencia general está asociada al conocimiento de la actividad empresarial en la industria en la que se compite (Zou \& Stan, 1998), mientras la experiencia internacional es indicativa del nivel de conocimiento sobre los mercados extranjeros (Fischer \& Reuber, 2003). La experiencia general proporciona una base fundamental para iniciar movimientos de internacionalización, fruto del aprendizaje organizacional y del aumento de la confianza directiva en la toma de decisiones (Majocchi, Bacchiocchi, \& Mayrhofer, 2005). Refuerza el nivel de planificación y reduce los niveles de improvisación, disminuyendo la probabilidad de tomar decisiones erróneas en mercados diferentes al doméstico (Nemkova, Souchon, \& Hughes, 2012). La experiencia internacional es generadora de un aprendizaje específico asociado a la actividad exportadora (Oura, Zilber, \& Lopez, 2016), proporcionando una valiosa información para facilitar la adaptación de la empresa a las necesidades de los mercados extranjeros, impulsando el posicionamiento internacional (Morgan et al., 2004). Este recurso de aprendizaje asociado a la experiencia -general e internacionalfomentará la capacidad de innovación de la empresa exportadora y reducirá los riesgos y barreras percibidas de la exportación, traduciéndose en un mayor espíritu emprendedor (Autio, Sapienza, \& Almeida, 2000; Knight \& Cavusgil, 2004). Todo ello impulsará la presencia internacional de la empresa exportadora, reflejándose positivamente en el grado, alcance e intensidad exportadora (Ruzo et al., 2011). Estos fundamentos nos llevan a plantear la siguiente hipótesis de investigación:

H2: Los recursos asociados a la experiencia, general e internacional, de la empresa exportadora influyen positivamente en el nivel de emprendimiento exportador.

Por otra parte, también el RBV en el contexto de la exportación refleja la existencia de una correlación positiva entre la creación y adaptación de sistemas e infraestructuras específicas para la actividad exportadora y el avance de la empresa en su proceso de internacionalización (Vermeulen \& Barkema, 2002). 
Así, la creación de un departamento de exportación ayuda a organizar y planificar la actividad exportadora (Caruana, Morris, \& Vella, 1998), al facilitar la captación de información de los mercados exteriores y agilizar la búsqueda y explotación de nuevas oportunidades. Ello aumenta la competitividad internacional de la empresa exportadora, reflejándose en su orientación emprendedora. Los argumentos expuestos nos llevan a plantear la siguiente hipótesis:

H3: Los recursos asociados a la existencia de una estructura específica -departamento de exportación- para la actividad exportadora influyen positivamente en el nivel de emprendimiento exportador.

\section{Emprendimiento y resultado de la actividad exportadora}

El resultado exportador es el reflejo de las decisiones estratégicas adoptadas por la empresa exportadora, y constituye un aspecto esencial para los responsables de exportación en el ámbito internacional (Madsen, 1998). Es la consecuencia de la planificación y ejecución de la estrategia de marketing de exportación, tiene carácter multidimensional e incluye tanto medidas cuantitativas (ej.: ventas, rentabilidad) como cualitativas (ej.: logro de objetivos) (Zou, Taylor, \& Osland, 1998). En este último caso, se requiere tener en cuenta las percepciones directivas en torno a los resultados de la actividad exportadora (Lages et al., 2008). Además, para su evaluación es recomendable no hacerlo de forma transversal, adoptando una visión de corto plazo -medición en un solo año-, sino en varios años, adoptando una perspectiva longitudinal (Sousa, 2004).

Teniendo en cuenta los aspectos señalados, en la presente investigación el resultado exportador se evalúa como una combinación de indicadores cuantitativos -crecimiento de las ventas de exportación- y cualitativos -satisfacción directiva-, adoptando una perspectiva longitudinal-se miden los resultados de exportación de los últimos tres años-y todo ello sin perder de vista las percepciones directivas sobre los resultados logrados.

En este contexto, algunos trabajos han mostrado que el alcance internacional de la empresa exportadora, evaluado a través del número de países en los cuales está presente, influye en el resultado exportador (Navarro, 2002; Ruzo et al., 2011). Así, se considera que aquellas empresas exportadoras que diversifican mercados, comercializando sus productos y/o servicios de forma simultánea en múltiples países, suelen alcanzar mejores resultados que aquellas otras cuyo alcance internacional es más limitado. Otros trabajos han demostrado que el desarrollo de procesos de internacionalización acelerado, lo cual suele ser indicativo de una mayor orientación internacional de la organización e incluso de una visión más global de los negocios internacionales, tiene una influencia positiva en los resultados alcanzados por la empresa en los mercados exteriores. Así se ha demostrado, desde la óptica de las exportaciones, en los trabajos de Zahra, Ireland y Hitt (2000), Kuivalainen et al. (2012) y Powell (2014), por ejemplo. También, algunos autores han señalado el positivo efecto que desempeña la intensidad exportadora de la organización en el resultado exportador (Ibeh, 2004; Nemkova et al., 2012). En definitiva, la literatura avala la posible influencia del emprendimiento exportador, definido por procesos de internacionalización acelerada, desarrollo de una estrategia de diversificación de mercados y una elevada intensidad exportadora, y los resultados empresariales logrados en los mercados extranjeros donde la empresa exportadora actúa. Estos argumentos apoyan el planteamiento de la siguiente hipótesis de investigación.

H4: Existe una relación positiva entre el nivel de emprendimiento de la empresa exportadora y sus resultados empresariales en los mercados exteriores.

Las relaciones propuestas se reflejan en la Figura 1.

\section{Figura 1. Modelo conceptual}

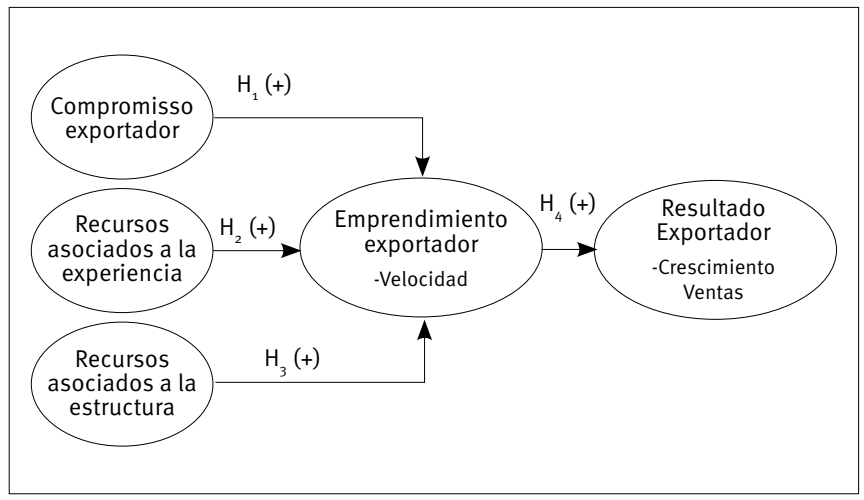

\section{MÉTODO}

A continuación, hacemos referencia a la muestra, la técnica de análisis de datos empleada y los referentes a las escalas de medidas.

\section{Muestra y técnica de análisis de datos}

Para contrastar las hipótesis planteadas se obtuvo, a partir de una base de datos del Instituto Español de Comercio Exterior (1.250 empresas exportadoras), una muestra de 212 empresas 
españolas que realizan operaciones de comercio exterior. La tasa de respuesta obtenida - $17 \%$ - puede considerarse aceptable (Menon, Bharadwaj, \& Howell, 1996). El cuestionario, enviado por e-mail, fue contestado por una única persona -responsable o director de exportación-. El empleo del método del informante único permite reducir errores y sesgos asociados a la tenencia de diferentes perspectivas de una misma cosa cuando se emplea más de un informante en cada empresa (Huber \& Power, 1985).

Las principales características de la muestra son: a) es de carácter multisectorial; b) la mayoría de las empresas incluidas son PYMEX -la media de empleados es de 37-; c) sólo un $33 \%$ dispone de una estructura específica-departamento de exportación- para las operaciones de comercio exterior; d) son empresas con una notable experiencia general -el $75 \%$ lleva más de 20 años en el sector- e internacional -el 60\% exporta desde hace más de 15 años-; e) la mayoría concentra su actividad en pocos mercados -el 70\% no exporta a más de cinco países-.

La modelización de ecuaciones estructurales usando PLS (partial least squares) fue la técnica empleada para el análisis de datos con el objeto de comprobar las relaciones entre los constructos, teniendo en cuenta las características del modelo propuesto (predictivo) y de la muestra -inferior a 250 observaciones- (Reinartz, Haenlein, \& Henseler, 2009). Específicamente, para el análisis empírico de datos se empleó el paquete estadístico SmartPLS 3.2.3.

\section{Escalas de medida}

Siguiendo las recomendaciones de diversos autores (Diamantopoulos, 2008; Diamantopoulos \& Siguaw, 2006; Diamantopoulos \& Winklhofer, 2001; Jarvis, Mackenzie, \& Podsakoff, 2003; Mackenzie, Podsakoff, \& Jarvis, 2005), en la presente investigación se ha empleado una doble perspectiva para definir las escalas de medida. Por un lado, consideramos el enfoque reflectivo, es decir, asumimos que los indicadores fluctúan acorde a las variaciones de la variable latente (Edwards \& Bagozzi, 2000) para definir el compromiso exportador y los recursos asociados a la experiencia y a la estructura. En este sentido, se concibe al compromiso exportador como la actitud -voluntad- de los responsables de la actividad exportadora de destinar adecuados recursos financieros, humanos y directivos a la exportación (Donthu \& Kim, 1993; Navarro et al., 2010a). En cuanto a los recursos, teniendo en cuenta el trabajo de Ruzo et al. (2011), se midió la experiencia general por los años transcurridos desde la creación de la empresa, la experiencia internacional por los años que la empresa lleva desarrollando operaciones de comercio exterior y los recursos asociados a la estructura por la disponibilidad o no de un departamento de exportación.
Por otro lado, teniendo en cuenta la complejidad de su medición y la distinta naturaleza de los indicadores asociados al emprendimiento exportador y al resultado de la actividad exportadora, se adoptó un enfoque formativo para definir cada uno de estos dos constructos, asumiendo que cambios en los indicadores generan variaciones en el concepto latente (Diamantopoulos, 1999). Ello supone que los constructos emprendimiento y resultado exportador quedan definidos como combinaciones -en nuestro caso, lineales- de sus indicadores más un término de error (Bollen, 1989; Bollen \& Lennox, 1991). En este contexto, se considera al resultado de la actividad exportadora como un constructo formativo de segundo orden, integrado por dos dimensiones de naturaleza muy distinta. La primera de las dimensiones es cuantitativa, definida por el crecimiento de las ventas de exportación en los últimos tres años (Cavusgil \& Zou, 1994; Navarro et al., 2010b). La segunda de las dimensiones es de tipo cualitativo, evaluada a través de la satisfacción percibida por los responsables de exportación asociada al logro de cinco objetivos en los últimos tres años (crecimiento de las ventas internacionales, imagen y notoriedad de la empresa en los mercados extranjeros, rentabilidad del negocio exportador, cuota de mercado y expansión internacional) (Cadogan, Diamantopoulos, \& Siguaw, 2002). El emprendimiento exportador también tiene un carácter formativo, pues queda configurado por tres dimensiones de muy distinta naturaleza: a) la velocidad de internacionalización, medida por el tiempo que la empresa tarda en iniciar su actividad exportadora (Acedo \& Jones, 2007); b) el grado o intensidad exportadora que, por ser indicativo del nivel de orientación hacia los mercados exteriores en relación al doméstico de la empresa exportadora (Kuivalainen et al., 2007), se midió por el ratio ventas de exportación respecto a las ventas totales; $c$ ) el alcance internacional, evaluado a través del número de mercados extranjeros -países- en los cuales la empresa exportadora genera ventas internacionales (BeleskaSpasova et al., 2012; Ruzo et al., 2011).

\section{RESULTADOS}

El análisis de los resultados incluye la evaluación del modelo de medida y los parámetros del modelo estructural, siguiendo las recomendaciones de Barclay, Higgins y Thompson, (1995). Para el modelo de medida se comenzó por asegurar la validez, convergente y discriminante, y la fiabilidad de las escalas de medidas, cumpliéndose para las escalas reflectivas (Tabla 1) que: a) las cargas factoriales fuesen superiores al valor recomendado de 0.70 (Carmines \& Zeller, 1979); b) los valores de la fiabilidad compuesta y de la varianza extraída media -AVE- superasen los 
límites recomendados de 0.7 y 0.5 , respectivamente (Fornell \& Larcker, 1981); c) las correlaciones (Tabla 2) entre cada par de constructos no excediesen el valor de la raíz cuadrada de AVE de cada constructo y que los ratios de correlaciones según el método HTMT (Henseler, Ringle, \& Sarstedt, 2015) no superasen el nivel crítico de 0.85 (Tabla 2). En el caso de las escalas formativas, se garantizó la ausencia de multicolinealidad, de forma que el factor de inflación de la varianza (FIV) fuese inferior al valor recomendado de 5 (Tabla 1) (Diamantopoulos \& Winklhofer, 2001).

\section{Tabla 1. Evaluación del modelo de medida}

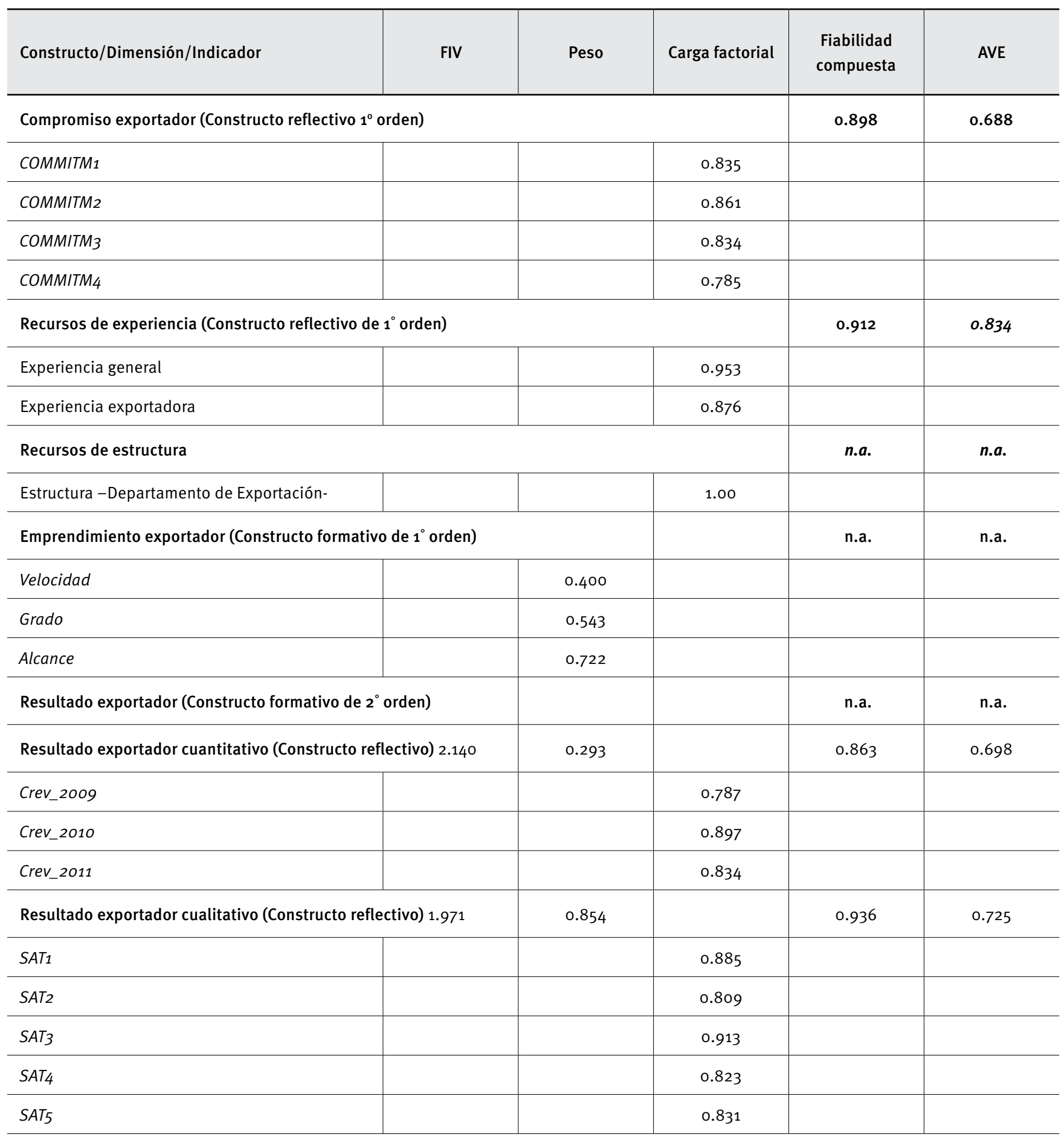

Nota: n.a. - no aplicable 
Tabla 2. Validez discriminante

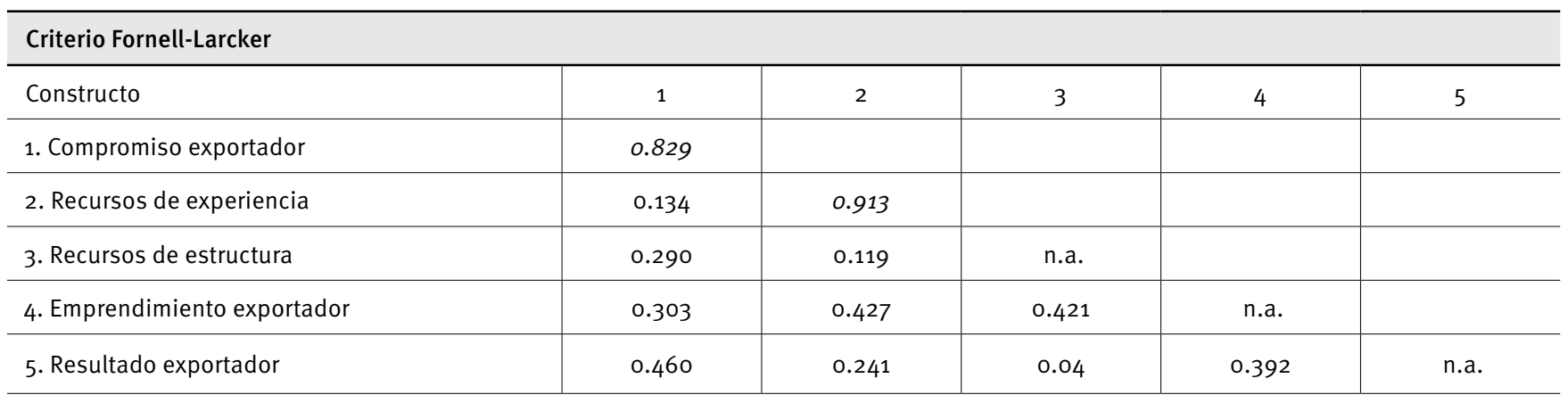

Nota: En la diagonal principal se recoge la raíz cuadrada de AVE; n.a.: No aplicable; Fuera de la diagonal principal se recogen las correlaciones entre constructos

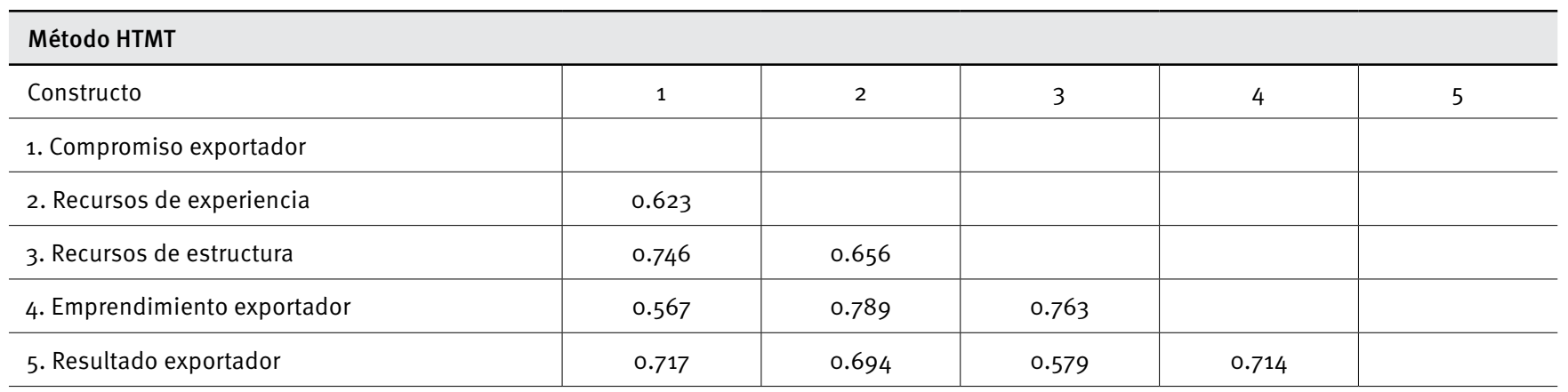

Una vez comprobadas las propiedades psicométricas de las escalas, se procedió al contraste de hipótesis. Para ello se empleó el método de remuestreo mediante bootstrap -100o submuestras (Davidson \& MacKinnon, 200o). Los parámetros estructurales (t-values y coeficientes $\beta$ ) que permiten aceptar o rechazar las hipótesis propuestas se recogen en la Tabla 3, aceptándose en la dirección propuesta las cuatro hipótesis planteadas. El modelo estructural se recoge de forma gráfica en la Figura 2.

\section{Tabla 3. Parámetros asociados al contraste de hipótesis}

\begin{tabular}{|c|c|c|c|}
\hline Hipótesis & $\beta$ & t-valor & Soportada \\
\hline $\mathrm{H}_{3}$ : Recursos de estructura - Emprendimiento exportador & 0.321 & $2.886^{\star \star \star}$ & $\mathrm{Si}$ \\
\hline $\mathrm{H}_{4}$ : Emprendimiento exportador - Resultado exportador & 0.393 & $2.896^{\star \star \star}$ & $\mathrm{Si}$ \\
\hline
\end{tabular}

Nota: $n s=$ no significativo (test de 1 -cola t(999) test) ${ }^{*} p<0.01 ;{ }^{* *} p<0.05 ;{ }^{* * *} p<0.1$

Los resultados reflejan que los factores internos considerados en la presente investigación -compromiso exportador, recursos asociados a la experiencia y a la estructura empresarial- logran explicar una varianza del emprendimiento exportador del 36,3\% $\left(R^{2}=0.363\right)$. Además, el emprendimiento exportador tiene una influencia decisiva en los resultados empresariales en los mercados internacionales, explicando por sí solo el $15,4 \%$ de la varianza del resultado exportador $\left(R^{2}=0.154\right)$.

Respecto a las hipótesis, señala que el compromiso exportador ejerce una influencia directa y positiva sobre el nivel de emprendimiento exportador, confirmando $\mathrm{H}_{1}\left(\beta_{1}=0.211\right.$; $\mathrm{t}$-value
$=2.074$ ). Ello puede ser debido a que el compromiso exportador atenúa los riesgos y barreras percibidas de la exportación (Styles \& Ambler, 2000), potenciando el desarrollo de una cultura orientada a los mercados exteriores que puede ser determinante de la velocidad, el grado y el alcance internacional de la organización (Navarro-García et al., 2013). Por otra parte, se corrobora que los recursos asociados al aprendizaje -experiencia-y la disposición de una estructura específica -departamento de exportación- en la empresa exportadora ejercen un efecto muy positivo sobre el emprendimiento exportador, confirmando $\mathrm{H}_{2}\left(\beta_{2}=0.383\right.$; t-value $=1.934) \mathrm{y} \mathrm{H}_{3}\left(\beta_{3}=0.321 ; \mathrm{t}\right.$-value $\left.=2.886\right)$. La disposición de estos 
recursos ofrece un mayor soporte en la toma de decisiones, aumentando la orientación de la empresa hacia los mercados internacionales (Ibeh, 2003). Finalmente, se demuestra el efecto directo y positivo que ejerce el nivel de emprendimiento en el resultado exportador (crecimiento de las ventas en los últimos tres años y satisfacción directiva), confirmando la hipótesis $\mathrm{H}_{4}\left(\beta_{4}=\right.$ 0.393; $\mathrm{t}$-value $=2.896$ ). Este resultado avala las conclusiones en torno a la nueva línea de investigación que viene desarrollándose en la literatura en los últimos años y que considera que aquellas empresas que inicien sus exportaciones en una fase temprana de su ciclo de vida, lo hagan de forma simultánea en numerosos países y con una notable intensidad, tendrán mayor probabilidad de lograr buenos resultados en los mercados exteriores (NavarroGarcía et al., 2015).

Figura 2. Modelo estructural

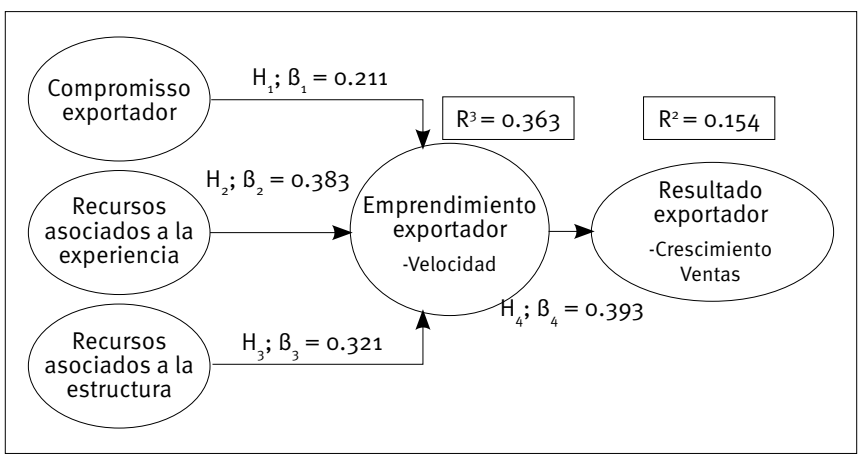

\section{CONCLUSIONES E IMPLICACIONES}

Las conclusiones del presente estudio se han dividido en dos partes diferenciadas acorde a los objetivos de la investigación. Así, se abordan en primer lugar las contribuciones desde el punto de vista académico y para la gestión empresarial, para exponer a continuación cuáles son las repercusiones de la investigación, asociadas al ámbito de la gestión pública, para un país como España, dado su perfil exportador.

Desde el punto de vista académico y científico, señala que se ha validado el modelo conceptual propuesto, considerando al emprendimiento exportador como un proceso asociado a los responsables de la actividad exportadora, a través del cual deciden cuándo (velocidad), cómo (grado) y dónde (alcance) la empresa va a desarrollar su actividad exportadora. En este proceso intervienen, siguiendo los fundamentos del RBV, factores de tipo interno, tanto de carácter personal asociados a las actitudes directivas -compromiso exportador-como de tipo organizacional, como son los recursos vinculados a la experiencia y a la estructura -departamento de exportación-. En este contexto, diversas son las conclusiones académicas que podemos ofrecer. Primero, las actitudes hacia la exportación que muestran los responsables de la actividad exportadora son determinantes esenciales del nivel de emprendimiento exportador. Esta afirmación está en línea con aportaciones previas que señalan que las actitudes directivas condicionan el proceso de internacionalización de las organizaciones (Lages et al., 2008), pudiendo influir en la orientación exportadora de la empresa (Acedo \& Galán, 2011). En nuestro caso, estas actitudes han sido medidas a través del compromiso exportador, entendido como la voluntad de la dirección de dedicar recursos humanos, financieros y directivos a su actividad exportadora.

Segundo, en conformidad con el RBV, los recursos de la empresa exportadora son impulsores -en nuestro caso, los principales- del emprendimiento exportador. Así, la experiencia general proporciona una base fundamental para iniciar movimientos de internacionalización, fruto del aprendizaje organizacional y del aumento de la confianza directiva en la toma de decisiones (Majocchi et al., 2005); refuerza el nivel de planificación y reduce los niveles de improvisación, disminuyendo la probabilidad de tomar decisiones erróneas en mercados diferentes al doméstico (Nemkova et al., 2012). Todo ello fomenta la orientación exportadora de la organización, impulsando el grado y alcance asociados al emprendimiento exportador (Ruzo et al., 2011). Por su parte, la experiencia internacional es generadora de un aprendizaje específico asociado a la actividad exportadora y proporciona una valiosa información para facilitar la adaptación de la empresa a las necesidades de los mercados extranjeros, facilitando el posicionamiento internacional (Morgan et al., 2004). Ello reduce los riesgos percibidos de la exportación, aumentando la orientación de la empresa hacia los mercados exteriores e impulsando su espíritu emprendedor (Autio et al., 2000; Knight \& Cavusgil, 2004). Por su parte, la existencia de un departamento de exportación ayuda a formalizar la toma de decisiones y aumenta los niveles de planificación (Caruana et al., 1998), incrementando la predisposición de la empresa exportadora a diversificar mercados (Ruzo et al., 2011).

Tercero, dado la existencia de una relación positiva entre el nivel de emprendimiento y el resultado exportador, es recomendable que las empresas exportadoras desarrollen procesos de internacionalización acelerados, aunque siempre con claras directrices estratégicas, planificando adecuadamente los tiempos y formas de entrada en los mercados extranjeros (Acedo \& Jones, 2007). Igualmente, es recomendable el desarrollo de estrategias de diversificación de mercados, plasmadas en un mayor alcance internacional de la organización, pues contribuye positivamente a mejorar el resultado exportador (Navarro, 2002; Ruzo et al., 2011). También lo hará la mayor orientación 
exportadora de la empresa, que se traducirá en una mayor participación de las ventas internacionales en las ventas totales de la organización (Kuivalainen et al., 2012).

En definitiva, desde el punto de vista académico, este trabajo contribuye significativamente a cubrir el importante gap existente en la literatura sobre emprendimiento y resultado exportador, de acuerdo con los fundamentos del RBV. Concretamente, este estudio demuestra que el nivel de emprendimiento de la empresa exportadora depende del compromiso que muestre la dirección con la exportación, y de los recursos asociados a la experiencia y estructura de la empresa exportadora. Por otra parte, los resultados del trabajo contribuyen a conocer cómo el nivel de emprendimiento exportador puede influir positivamente tanto en el crecimiento de las ventas internacionales a lo largo de un periodo de tiempo, como en la satisfacción que experimentan los directivos con el logro de objetivos en los mercados exteriores.

Desde la óptica de la gestión empresarial, el presente trabajo también ofrece importantes contribuciones que pueden ayudar a los directivos responsables de exportación a sistematizar la toma de decisiones. Así, la dirección de la empresa exportadora debe mostrar una actitud proactiva hacia los mercados exteriores, destinando los recursos humanos, financieros y directivos necesarios. Este compromiso exportador incentiva a la empresa exportadora a acelerar su proceso de internacionalización, diversificar mercados en la búsqueda de oportunidades comerciales y a mostrar una mayor intensidad exportadora. Igualmente, las empresas exportadoras deben ser proclives a la creación de estructuras específicas para la toma de decisiones, por ejemplo un departamento de exportación, pues ello facilitará la planificación de las exportaciones y agilizará la toma de decisiones. Todo ello redundará en un aumento de la orientación emprendedora de la organización. También lo hará el aprendizaje, fruto de la experiencia, tanto en la industria como específicamente en los mercados extranjeros. En este sentido, para lograr cuanto antes la necesaria experiencia internacional, es recomendable que la empresa exportadora acelere su proceso de internacionalización. Todo ello influirá positivamente en el resultado exportador.

Ahora bien, desde la vertiente de las políticas públicas ¿Cuáles son las principales implicaciones del estudio para España, dado el perfil exportador de este país? El Gobierno de España, dentro del Plan Estratégico de Internacionalización de la Economía Española 2014-2015, ha establecido como epicentro de su actuación la mejora de la competitividad del sector exterior (principal motor de salida de la crisis que vive España en los últimos años), de forma que contribuya al crecimiento y a la creación de empleo, considerándose a la exportación como vía preferente de acceso a los mercados internacionales. Se pretende diseñar estrategias de medio y largo plazo que incidan sobre tres aspectos básicos: a) impulsar el acceso a los mercados extranjeros de las empresas españolas con potencial exportador; b) lograr que los exportadores esporádicos se conviertan en exportadores regulares; c) aumentar la propensión a exportary la base exportadora de la economía española, diversificando los mercados de destino de las exportaciones. Estos tres aspectos los ha resumido bajo el objetivo estratégico y prioritario de "fomentar el emprendimiento exportador como factor de estabilidad y crecimiento económico". En este sentido, el Gobierno de España, considera necesario el desarrollo de estudios, como el planteado en la presente investigación, que aporten evidencias empíricas de qué factores concretos alientan la cultura emprendedora de las empresas exportadoras españolas y qué consecuencias pueden tener en los resultados internacionales. Ello ayudará al desarrollo de políticas públicas que resulten eficaces en la mejora de la competitividad exportadora de la economía española. Por ello, desde la óptica pública, la investigación desarrollada aporta una manera novedosa de evaluar el emprendimiento exportador, que puede ser empleada por el Gobierno de España en sus Planes Estratégicos de Internacionalización, integrando tres dimensiones básicas: velocidad, grado o alcance internacional, e intensidad o propensión a exportar. Otra contribución del estudio, asociada a la ansiada mejora de la cultura emprendedora de las empresas exportadoras por parte del Gobierno de España, es el desarrollo de actitudes favorables a la internacionalización, como ocurre en nuestro caso con el compromiso exportador, aspecto que debe ser fomentado con medidas concretas de concienciación directiva de la importancia para España de la exportación. Así se contempla en el reciente lanzamiento de la denominada "Ventana Global de Exportación e Inversiones" que ha puesto en marcha el ICEX, la cual debe favorecer el desarrollo de recursos y capacidades orientados a la exportación, como los asociados al aprendizaje y gestión del conocimiento de los mercados extranjeros. Estos aspectos están en línea con lo apuntado en la presente investigación, en lo referente a los recursos vinculados a la experiencia y a la estructura -departamento de exportación-.

En definitiva, el Gobierno de España espera, en el citado Plan Estratégico de Internacionalización 2014-2015, que el fomento del emprendimiento exportador de las empresas españolas redunde en una mejora de los resultados internacionales. Así se demuestra en la investigación desarrollada, evaluando el resultado exportador no sólo desde una vertiente cuantitativa (ventas, crecimiento de las ventas, rentabilidad) sino también cualitativa (satisfacción directiva). La mejora del emprendimiento exportador se considera especialmente importante en el caso de 
las PYMEX, pues, como señala el Gobierno de España, el proceso de internacionalización de la economía española adolece de ciertas debilidades que deben ir corrigiéndose, entre ellas, la concentración de las exportaciones en un número reducido de empresas, normalmente de gran tamaño y la escasa la propensión a exportar de las PYMEX. Así, en 2013, las empresas de menor volumen de exportación, inferior a 25.000 euros anuales, suponen el $69,4 \%$ del número de empresas exportadoras y representan tan sólo el o,1\% de la exportación total. La corrección de esta debilidad está conllevando el desarrollo de medidas específicas para las PYMEX españolas, como son las incluidas en el Programa ICEX-NEXT, donde se pretende impulsar el compromiso de recursos -humanos, financieros y directivos- hacia la actividad exportadora de las PYMEX españolas, pues fortalecerá su competitividad internacional y, con ella, la de la economía española. En este sentido, los resultados de la investigación desarrollada pueden servir como eje para la toma de decisiones en el ámbito de la gestión pública, pues de las 212 empresas analizas en la muestra, el $92 \%$ (196 empresas) son PYMEX.

\section{LIMITACIONES Y FUTURAS LÍNEAS DE INVESTIGACIÓN}

Aunque las contribuciones científicas y profesionales de la presente investigación sean notables, esta no está exenta de limitaciones que constituyen retos futuros que deben abordarse. Tres limitaciones pueden señalarse al respecto: a) la naturaleza del estudio es principalmente transversal, tomándose información de los responsables de exportación en un determinado momento, lo que impide obtener una visión más amplia de la influencia a medio y largo plazo de los recursos empresariales y del compromiso exportador en los niveles de emprendimiento internacional y, por ende, en los resultados empresariales. Estudios de tipo longitudinal permitirán superar la debilidad apuntada; b) los resultados obtenidos no son generalizables, pues la investigación se ha realizado con una muestra de empresas de un solo país, España. Para corregir esta limitación, deberán desarrollarse estudios multipaís y de carácter cross-cultural; c) el modelo propuesto es una representación simplificada de la realidad que no ha tenido en cuenta todas las posibles variables que pueden influir en el emprendimiento exportador y en el resultado de la actividad exportadora. Por ello, la inclusión de otras variables -naturaleza del producto exportado, sector de actividad, calidad de las relaciones con los distribuidores internacionales, etc.- puede contribuir a explicar la varianza de los constructos considerados como dependientes en el modelo.

\section{REFERENCIAS}

Aaby, N. E., \& Slater, S. F. (1989). Management influences on export performance: A review of the empirical literature 1978-1988. International Marketing Review, 6(4), 7-26. doi:10.1108/EUMoo00000001516

Acedo, F. J., \& Galán, J. L. (2011). Export stimuli revisited: The influence of the characteristics of managerial decision makers on international behaviour. International Small Business Journal, 29(6), 648-670. doi:10.1177/0266242610375771

Acedo, F. J., \& Jones, M. V. (2007). Speed of internationalization and entrepreneurial cognition: Insights and a comparison between international new ventures, exporters and domestic firms. Journal of World Business, 42(3), 236-252. doi:10.1016/j.jwb.2007.04.012

Alteren, G., \& Tudoran, A. A. (2016). Enhancing export performance: Betting on customer orientation, behavioral commitment, and communication. International Business Review, 25(1), 370-381. doi:10.1016/j. ibusrev.2015.07.004

Audretsch, D. B., \& Keilbach, M. (2004). Entrepreneurship capital and economic performance. Regional Studies, 38(8), 949-959. doi:10.1080/0034340042000280956

Autio, E., Sapienza, H. J., \& Almeida, J. G. (2000). Effects of age at entry, knowledge intensity, and imitability on international growth. Academy of Management Journal, 43(5), 909-924.

Barclay, D., Higgins, C., \& Thompson, R. L. (1995). The partial least squares (PLS) approach to causal modelling: Personal computer adoption and use as an illustration. Technology Studies, 2(2), 285309.

Barney, J. (1991). Firm resources and sustained competitive advantage. Journal of Management, 17(1), 99-120. doi:10.1177/014920639101700108

Beamish, P. W., Karavis, L., Goerzen, A., \& Lane, C. (1999). The relationship between organizational structure and export performance. Management International Review, 39(1), 37-54.

Beleska-Spasova, E., Glaister, K. W., \& Stride, C. (2012). Resource determinants of strategy and performance: The case of British exporters. Journal of World Business, 47(4), 635-647. doi:10.1016/j. jwb.2011.09.001

Blalock, G. \& Gertler, P. J. (2004). Learning from exporting revisited in a less developed setting. Journal of Development Economics, 75(2), 397-416. doi:10.1016/j.jdevec0.2004.06.004

Bollen, K. (1989). Structural equations with latent variables. New York, EEUU: John Wiley y Sons, Inc.

Bollen, K., \& Lennox, R. (1991). Conventional wisdom on measurement: A structural equation perspective. Psychological Paradigm, 110(2), 305-314. doi:10.1037/0033-2909.110.2.305

Cadogan, J. W., Diamantopoulos, A., \& Siguaw J. A. (2002). Export market-oriented activities: Their antecedents and performance consequences. Journal of International Business Studies, 33(3), 615-626.

Cadogan, J. W., Kuivalainen, O., \& Sundqvist, S. (2009). Export market-oriented behavior and export performance: Quadratic and moderating effects under differing degrees of market dynamism and internationalization. Journal of International Marketing, 17(4), 71-89. doi:10.1509/jimk.17.4.71

Carmines, E. G., \& Zeller, R. A. (1979). Reliability and validity assessment. Beverly Hills, EEUU: Sage. 
Caruana, A., Morris, M., \& Vella, A. (1998). The effect of centralization and formalization on entrepreneurship in export firms. Journal of Small Business Management, 36(1), 16-29.

Cavusgil, S. T., \& Zou, S. (1994). Marketing strategy-performance relationship: An investigation of the empirical link in export market ventures. Journal of Marketing, 58(1), 1-21. doi:10.2307/1252247

Colton, D. A., Roth, M. S., \& Bearden, W. O. (2010). Drivers of international e-tail performance: The complexities of orientations and resources. Journal of International Marketing, 18(1), 1-22. doi:10.1509/ jimk.18.1.1

Davidson, R., \& MacKinnon, J. G. (2000). Bootstrap tests: How many bootstraps? Econometric Reviews, 19(1), 55-68. doi:10.1080/07474930008800459

Dhanaraj, C., \& Beamish, P. W. (2003). A resource-based approach to the study of export performance. Journal of Small Business Manage ment, 41(3), 242-261. doi:10.1111/1540-627x.00080

Diamantopoulos, A. (1999). Viewpoint -Export performance measurement: Reflective versus formative indicators. International Marketing Review, 16(6), 444-457. doi:10.1108/02651339910300422

Diamantopoulos, A. (2008). Formative indicators: Introduction to the special issue. Journal of Business Research, 61(12), 1201-1202. doi:10.1016/j.jbusres.2008.01.008

Diamantopoulos, A., \& Siguaw, J. A. (2006). Formative versus reflective indicators in organizational measure development: A comparison and empirical illustration. British Journal of Management, 17(4), 263 282. doi:10.1111/j.1467-8551.2006.00500.x

Diamantopoulos, A., \& Winklhofer, H. M. (2001). Index construction with formative indicators: An alternative to scale development. Journal of Marketing Research, 38(2), 269-277.

Donthu, N., \& Kim, S. H. (1993). Implications of firm controllable factors on export growth. Journal of Global Marketing, 7(1), 47-64. doi:10.1300/Jo42v07no1_04

Edwards, J. R., \& Bagozzi, R. P. (2000). On the nature and direction of relationships between construct and measures. Psychological Meth ods, 5(2), 155-174. doi:10.1037/1082-989X.5.2.155

Fischer, E., \& Reuber, A. R. (2003). Targeting export support to SMEs: Owners' international experience as a segmentation basis. Small Business Economics, 20(1), 69-82. doi:10.1023/a:1020296405149

Fornell, C., \& Larcker, D.F. (1981). Evaluating structural equation models with unobservable variables and measurement error. Journal of Marketing Research, 18(1), 39-50. doi:10.2307/3151312

Gallego, M. Á., \& Casillas, J. C. (2014). Choice of markets for initial export activities: Differences between early and late exporters. International Business Review, 23(5), 1021-1033. doi:10.1016/j.ibusrev.2014.03.004

Hessels, S. J. A. (2007). Innovation and international involvement of Dutch SMEs. International Journal of Entrepreneurship and Small Business, 4(3), 234-255. doi:10.1504/IJESB.2007.013250

Hessels, S. J. A., \& van Stel, A. van. (2011). Entrepreneurship, export orientation, and economic growth. Small Business Economics, 37(2), 255-268., doi:10.1007/s11187-009-9233-3

Huber, G. P., \& Power, D. J. (1985). Retrospective reports of strategic-level managers: Guidelines for increasing their accuracy. Strategic Management Journal, 6(2), 171-180. doi:10.1002/smj.4250060206

Hultman, M., Robson, M. J., \& Katsikeas, C. S. (2009). Export product strategy fit and performance: An empirical investigation. Journal of International Marketing, 17(4), 1-23. doi:10.1509/jimk.17.4.1

Ibeh, K. I. (2003). Toward a contingency framework of export entrepreneurship: Conceptualisations and empirical evidence. Small Business Economics, 20(1), 49-68.
Ibeh, K. I. (2004). Furthering export participation in less performing developing countries: The effects of entrepreneurial orientation and managerial capacity factors. International Journal of Social Economics, 31(1/2), 94-110. doi:10.1108/03068290410515448

Ibeh, K. I., \& Young, S. (2001). Exporting as an entrepreneurial act: An empirical study of Nigerian firms. European Journal of Marketing, 35(5/6), 566-586. doi:10.1108/03090560110388114

Henseler, J., Ringle, C. M., \& Sarstedt, M. (2015). A new criterion for assessing discriminant validity in variance-based structural equation modeling. Journal of the Academy of Marketing Science, 43(1), 115135. doi:10.1007/s11747-014-0403-8

Jarvis, C. B., Mackenzie, S. B., \& Podsakoff, P. M. (2003). A critical review of construct indicators and measurement model misspecification in marketing and consumer research. Journal of Consumer Research, 30(2), 199-218. doi:10.1086/376806

Jones, M. V., Coviello, N., \& Tang, Y. K. (2011). International entrepreneurship research (1989-2009): A domain ontology and thematic analysis. Journal of Business Venturing, 26(6), 632-659. doi:10.1016/j. jbusvent.2011.04.001

Keupp, M. M., \& Gassmann, O. (2009). The past and the future of international entrepreneurship: A review and suggestions for developing the field. Journal of Management, 35(3), 600-633. doi:10.1177/0149206308330558

Knight, G. A., \& Cavusgil, S. T. (2004). Innovation, organizational capabilities, and the born-global firm. Journal of International Business Studies, 35(2), 124-141

Knight, G. A., \& Liesch, P. W. (2016). Internationalization: From incremental to born global. Journal of World Business, 51(1), 93-102. doi:10.1016/j.jwb.2015.08.011

Kuivalainen, O., Saarenketo, S., \& Puumalainen, K. (2012). Start-up patterns of internationalization: A framework and its application in the context of knowledge-intensive SMEs. European Management Journal, 30(4), 372-385. doi:10.1016/j.emj.2012.01.001

Kuivalainen, O., Sundqvist, S., \& Servais, P. (2007). Firms' degree of born-globalness, international entrepreneurial orientation and export performance. Journal of World Business, 42(3), 253-267. doi:10.1016/j.jwb.2007.04.010

Lages, L. F., Jap, S. D., \& Griffith, D. A. (2008). The role of past performance in export ventures: A short-term reactive approach. Journal of International Business Studies, 39(2), 304-325. doi:10.1057/palgrave.jibs. 8400339

Lages, L. F., \& Montgomery, D. B. (2004). Export performance as an antecedent of export commitment and marketing strategy adaptation: Evidence from small and medium-sized exporters. European Journal of Marketing, 38(9/10), 1186-1214. doi:10.1108/03090560410548933

Lages, L. F., Silva, G. \& Styles, C. (2009). Relationship capabilities, quality, and innovation as determinants of export performance. Journal of International Marketing, 17(4), 47-70. doi:10.1509/jimk.17.4.47

Machado, M. A., Nique, W. M., \& Fehse, F. B. (2016). International orientation and export commitment in fast small and medium size firms internationalization: Scales validation and implications for the Brazilian case. Revista de Administração da Universidade de São Paulo, 51(3), 255-265. doi:10.1016/j.rausp.2016.06.002

Mackenzie, S. B., Podsakoff, P. M., \& Jarvis, C. B. (2005). The problem of measurement model misspecification in behavioural and organizational research and some recommended solutions. Journal of Applied Psychology, 9o(4), 710-730. doi:10.1037/0021-9010.90.4.710 
Madsen, T. K. (1998). Executive insights: Managerial judgment of export performance. Journal of International Marketing, 6(3), 82-93.

Majocchi, A., Bacchiocchi, E., \& Mayrhofer, U. (2005). Firm size, business experience and export intensity in SMEs: A longitudinal approach to complex relationships. International Business Review, 14(6), 719-738. doi:10.1016/j.ibusrev.2005.07.004

Makadok, R. (2001). Toward a synthesis of the resource-based and dynamic-capability views of rent creation. Strategic Management Journal, 22(5), 387-401. doi:10.1002/smj.158

Menon, A., Bharadwaj, S. G., \& Howell, R. D. (1996). The quality and effectiveness of marketing strategy: Effect of functional and dysfunctional conflict in intraorganizational relationships. Journal of Academy of Marketing Science, 24(4), 299-313. doi:10.1177/0092070396244002

Morgan, N. A., Kaleka, A., \& Katsikeas, C. S. (2004). Antecedents of export ventures performance: A theoretical model and empirical assessment. Journal of Marketing, 68(1), 90-108.

Morgan, N. A., Vorhies, D. W., \& Schlegelmilch, B. B. (2006). Resource-performance relationships in industrial export ventures: The role of resource inimitability and substitutability. Industrial Marketing Management, 35(5), 621-633. doi:10.1016/j.indmarman.2005.05.018

Navarro, A. (2002). Efecto de la estrategia de expansión exterior sobre el resultado exportador de la organización. Información Comercial Española, (802), 99-115.

Navarro, A., Losada, F., Ruzo, E., \& Díez, J. A. (2010a). Implications of perceived competitive advantages, adaptation of marketing tactics and export commitment on export performance. Journal of World Business, 45(1), 49-58. doi:10.1016/j.jwb.2009.04.004

Navarro, A., Acedo, F. J., Robson, M. J., Ruzo, E., \& Losada, F. (2010b). Antecedents and consequences of firms' export commitment: An empirical study. Journal of International Marketing, 18(3), 41-61. doi: 10.1509/jimk.18.3.41

Navarro, A., Acedo, F. J., Losada, F., \& Ruzo, E. (2011). Integrated model of export activity: Analysis of heterogeneity in managers' orientations and perceptions on strategic marketing management in foreign markets. Journal of Marketing Theory and Practice, 19(2), 187-204. doi: 10.2753/MTP1069-6679190205

Navarro-Garcia, A., Rondán-Cataluña, F. J., \& Acedo-González, F. J. (2013). The importance of an export-oriented culture for export performance. European Journal of International Management, 7(3), 254277. doi: 10.1504/EJIM.2013.054325

Navarro-García, A. (2016). Drivers of export entrepreneurship. International Business Review, 25(1), 244-254. doi:10.1016/j.ibusrev.2015.05.007

Navarro-García, A., Schmidt, A. C. M., \& Rey-Moreno, M. (2015). Antecedents and consequences of export entrepreneurship. Journal of Business Research, 68(7), 1532-1538. doi:10.1016/j.jbusres.2015.01.047

Nemkova, E., Souchon, A. L., \& Hughes, P. (2012). Export decision-making orientation: An exploratory study. International Marketing Review, 29(4), 349-378. doi:10.1108/02651331211242610

Oura, M. M., Zilber, S. N., \& Lopes, E. L. (2016). Innovation capacity, international experience and export performance of SMEs in Brazil. International Business Review, 25(4), 921-932. doi:10.1016/j.ibusrev.2015.12.002
Pla-Barber, J., \& Alegre, J. (2007). Analysing the link between export intensity, innovation and firm size in a science-based industry. International Business Review, 16(3), 275-293. doi:10.1016/j.ibusrev.2007.02.005

Powell, K. S. (2014). Profitability and speed of foreign market entry. Management International Review, 54(1), 31-45. doi:10.1007/s11575013-0184-1

Reinartz, W., Haenlein, M., \& Henseler, J. (2009). An empirical comparison of the efficacy of covariance-based and variance-based SEM. International Journal of Research in Marketing, 26(4), 332-344. doi:10.1016/j.ijresmar.2009.08.001

Ruzo, E., Losada, F., Navarro, A., \& Díez, J. A. (2011). Resources and international marketing strategy in export firms: Implications for export performance. Management Research Review, 34(5), 496-518. doi:10.1108/01409171111128698

Singh, D. A. (2009). Export performance of emerging market firms. International Business Review, 18(4), 321-330. doi:10.1016/j.ibusrev.2009.03.002

Sousa, C. M., Martínez-López, F. J., \& Coelho, F. (2008). The determinants of export performance: A review of the research in the literature between 1998 and 2005. International Journal of Management Reviews, 10 (4), 343-374. doi:10.1111/j.1468-2370.2008.00232.x

Sousa, C. M. P. (2004). Export performance measurement: An evaluation of the empirical research in the literature. Academy of Marketing Science Review, 9(12), 1-23.

Styles, C., \& Ambler, T. (2000). The impact of relational variables on export performance: An empirical investigation in Australia and the UK. Australian Journal of Management, 25(3), 261-281. doi:10.1177/031289620002500302

Vermeulen, F., \& Barkema, H. (2002). Pace, rhythm, and scope: Process dependence in building a profitable multinational corporation. Strategic Management Journal, 23(7), 637-653. doi:10.1002/smj.243

Yeoh, P. L. (2004). International learning: Antecedents and performance implications among newly internationalizing companies in an exporting context. International Marketing Review, 21(4/5), 511-535. doi:10.1108/02651330410547171

Yeoh, P. L., \& Jeong, I. (1995). Contingency relationships between entrepreneurship, export channel structure and environment: A proposed conceptual model of export performance. European Journal of Marketing, 29(8), 95-115. doi:10.1108/03090569510097574

Zahra, S. A., Ireland, R. D., \& Hitt, M.A. (2000). International expansion by new venture firms: International diversity, mode of market entry, technological learning, and performance. Academy of Management Journal, 43(5), 925-950. doi:10.2307/1556420

Zander, I., McDougall-Covin, P., \& Rose, E. L. (2015). Born globals and international business: Evolution of a field of research. Journal of International Business Studies, 46(1), 27-35. doi:10.1057/jibs.2014.60

Zou, S., \& Stan, S. (1998). The determinants of export performance: A review of the empirical literature between 1987 and 1997. International Marketing Review, 15(5), 333-356. doi:10.1108/02651339810236290

Zou, S., Taylor, C. R., \& Osland, G. E. (1998). The EXPERF scale: A cross-national generalized export performance measure. Journal of International Marketing, 6(3), 37-58. 